\title{
The Gravitational Horizon for a Universe with Phantom Energy
}

\section{Fulvio Melia ${ }^{1}$}

Department of Physics, the Applied Math Program, and Steward Observatory

The University of Arizona

Tucson, AZ 85721

E-mail: fmelia@as.arizona.edu

\begin{abstract}
The Universe has a gravitational horizon, coincident with the Hubble sphere, that plays an important role in how we interpret the cosmological data. Recently, however, its significance as a true horizon has been called into question, even for cosmologies with an equation-of-state $w \equiv p / \rho \geq-1$, where $p$ and $\rho$ are the total pressure and energy density, respectively. The claim behind this argument is that its radius $R_{\mathrm{h}}$ does not constitute a limit to our observability when the Universe contains phantom energy, i.e., when $w<-1$, as if somehow that mitigates the relevance of $R_{\mathrm{h}}$ to the observations when $w \geq-1$. In this paper, we reaffirm the role of $R_{\mathrm{h}}$ as the limit to how far we can see sources in the cosmos, regardless of the Universe's equation of state, and point out that claims to the contrary are simply based on an improper interpretation of the null geodesics.
\end{abstract}

\footnotetext{
${ }^{1}$ John Woodruff Simpson Fellow.
} 


\section{Contents}

1 Introduction $\quad 1$

2 The Gravitational (Cosmic) Horizon $r$

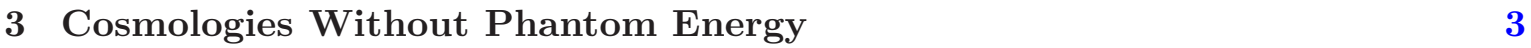

4 Phantom Energy 4

5 Concluding Remarks $\quad 7$

\section{Introduction}

A newly recognized horizon (dubbed the "Cosmic Horizon" in [1]) is beginning to play a crucial role in our understanding of how the Universe evolves. The distance to this horizon is the Universe's gravitational radius, i.e., the radius $R_{\mathrm{h}}$ at which a proper sphere encloses sufficient mass-energy to turn it into a Schwarzschild surface for an observer at the origin of the coordinates (see also [2]). Regardless of which specific cosmological model one adopts, $R_{\mathrm{h}}$ is always given by the expression

$$
R_{\mathrm{h}}=\frac{2 G M\left(R_{\mathrm{h}}\right)}{c^{2}}
$$

where $M\left(R_{\mathrm{h}}\right)=(4 \pi / 3) R_{\mathrm{h}}^{3} \rho / c^{2}$, in terms of the total energy density $\rho$. Thus, $R_{\mathrm{h}}$ is given as $\left(3 c^{4} / 8 \pi G \rho\right)^{1 / 2}$ which, for a flat universe, may also be written more simply as $R_{\mathrm{h}}(t)=c / H(t)$, where $H(t)$ is the (time-dependent) Hubble constant.

Although not defined in this fashion, the Hubble radius $c / H(t)$ is therefore clearly a manifestation of the gravitational horizon $R_{\mathrm{h}}$ emerging directly from the Robertson-Walker metric written in terms of the proper distance $R=a(t) r$, the expansion factor $a(t)$, and co-moving radius $r$. What this means, of course, is that the Hubble sphere is not merely an empirical artifact of the expanding universe, but actually encloses a volume centered on the observer containing sufficient mass-energy for its surface to function as a static horizon. That also means that the speed of expansion a distance $R_{\mathrm{h}}$ away from us must be equal to $c$, just as the speed of matter falling into a compact object reaches $c$ at the black hole's horizon - and this is in fact the criterion used to define the Hubble radius in the first place. Very importantly, it may also help the reader to know that since the gravitational radius coincides identically with the Hubble radius, all of the known equations and constraints (e.g., 2.4 below) satisfied by the latter must also be satisfied by the former.

Since its introduction, however, some confusion regarding the properties of $R_{\mathrm{h}}$ has crept into the literature, due to a misunderstanding of the role it plays in our observations. For example, it has sometimes been suggested (see, e.g., the earlier paper [3], and the more recent work [4]) that sources beyond $R_{\mathrm{h}}\left(t_{0}\right)$ are observable today (at cosmic time $t_{0}$ ), which is certainly not the case. To eliminate misconceptions such as this, some effort was made [5] to elaborate upon what the gravitational radius $R_{\mathrm{h}}$ is - and what it is not. Though first defined in [1], an unrecognized form of $R_{\mathrm{h}}$ actually appeared in de Sitter's own account of his 
spacetime metric [6]. In reference [5], photon trajectories were calculated for various wellstudied Friedmann-Robertson-Walker (FRW) cosmologies, including $\Lambda$ CDM, demonstrating that the null geodesics reaching us at $t_{0}$ have never crossed $R_{\mathrm{h}}\left(t_{0}\right)$. (To be very precise, what we mean by this is that the proper distance $R_{\gamma}$ of photons travelling along these null geodesics never attained a value exceeding $R_{\mathrm{h}}\left[t_{0}\right]$.) More generally — and more conclusively they proved that $R_{\mathrm{h}}\left(t_{0}\right)$ is a real limit to our observability for any cosmology that does not contain phantom energy [7], i.e., any cosmology whose equation of state is $p \geq-\rho$, in terms of the total pressure $p$ and total energy density $\rho$. (This is a very simple proof and, for completeness, we will reproduce it in the discussion below.)

In spite of this, the issue has been revisited [8] with the argument that a Universe with phantom energy violates this limit, thereby demoting $R_{\mathrm{h}}$ from its acknowledgment as a true horizon. Of course, even if it were true that null geodesics could reach us from beyond $R_{\mathrm{h}}$ when $p<-\rho$, that would simply be highlighting a peculiarity of phantom cosmologies, which have other interesting features, such as the acausal transfer of energy. But even in that scenario, it would have nothing to say about the role played by $R_{\mathrm{h}}$ in delimiting what we can see in any cosmology that does not contain phantom energy. The purpose of this paper is to reiterate what the physical meaning of the Universe's gravitational radius is and to demonstrate that, even in cases where $p<-\rho$, no null geodesics reaching us have ever crossed the maximum extent of our cosmic horizon.

\section{The Gravitational (Cosmic) Horizon}

Standard cosmology is based on the Friedmann-Robertson-Walker (FRW) metric for a spatially homogeneous and isotropic three-dimensional space. In terms of the proper time $t$ measured by a comoving observer, and the corresponding radial $(r)$ and angular coordinates $(\theta$ and $\phi)$ in the comoving frame, an interval $d s$ in this metric is written as

$$
d s^{2}=c^{2} d t^{2}-a^{2}(t)\left[d r^{2}\left(1-k r^{2}\right)^{-1}+r^{2}\left(d \theta^{2}+\sin ^{2} \theta d \phi^{2}\right)\right],
$$

where $a(t)$ is the aforementioned expansion factor and the constant $k$ is +1 for a closed universe, 0 for a flat, open universe, or -1 for an open universe.

The FRW metric may also be written in terms of the proper distance $R[1,9,10]$, and whereas $(c t, r, \theta, \phi)$ describe events in a frame "falling" freely with the cosmic expansion, the second set of coordinates are referenced to a particular individual who describes the spacetime relative to the origin at his location. For a flat universe $(k=0)$, it is not difficult to show that

$$
d s^{2}=\Phi\left[c d t+\left(\frac{R}{R_{\mathrm{h}}}\right) \Phi^{-1} d R\right]^{2}-\Phi^{-1} d R^{2}-R^{2} d \Omega^{2},
$$

where the function

$$
\Phi \equiv 1-\left(\frac{R}{R_{\mathrm{h}}}\right)^{2}
$$

signals the dependence of the metric on the proximity of the proper radius $R$ to the gravitational radius $R_{\mathrm{h}}$. The exact form of $R_{\mathrm{h}}$ depends on the composition of the Universe. For example, in de Sitter space (which contains only a cosmological constant $\Lambda$ ), $R_{\mathrm{h}}=c / H_{0}$, in terms of the (time-invariant) Hubble constant $H_{0}$. It is equal to $2 c t$ in a radiation-dominated universe and $3 c t / 2$ when the Universe contains only (visible and dark) matter. 
The reason why $R_{\mathrm{h}}$ is an essential ingredient of the metric written in the form of Equation (2.2) can be understood in the context of the Birkhoff theorem [11] and its corollary. ${ }^{1}$ Yet the physical meaning of $R_{\mathrm{h}}$ is still elusive to many, possibly because of the widely held belief that all horizons must necessarily be asymptotic surfaces attained when $t \rightarrow \infty$. The so-called event horizon (see [12]) is indeed of this type, representing the ultimate limit to our observability to the end of time. However, $R_{\mathrm{h}}$ is not in this category - nor should it be. Unlike the event horizon, the gravitational radius is a time-dependent quantity that increases in value at a rate determined by the evolving constituents of the Universe, specifically, the value of the equation-of-state parameter $w$, defined by the relation $p=w \rho$. For some cosmologies, $R_{\mathrm{h}}$ may turn into the event horizon when the cosmic time approaches infinity.

It is not difficult to see that the evolution of $R_{\mathrm{h}}$ is given by the equation

$$
\dot{R}_{\mathrm{h}}=\frac{3}{2}(1+w) c
$$

This is easy to demonstrate from the definition of $R_{\mathrm{h}}$ in Equation (2.1) and the Friedmann equation. But as we mentioned earlier, it is also known that the Hubble radius satisfies this equation, so the gravitational radius must satisfy it as well. Clearly, $R_{\mathrm{h}}$ is constant only for the de Sitter metric, where $w=-1$ and therefore $\dot{R}_{\mathrm{h}}=0$. For all other values of $w>-1$, $\dot{R}_{\mathrm{h}}>0$. So in $\Lambda \mathrm{CDM}$, for example, where the Universe is currently dominated by a blend of matter and dark energy, $\dot{R}_{\mathrm{h}}>0$. If dark energy were a cosmological constant, however, the Universe would eventually become de Sitter as the density of matter drops to zero, and we would therefore expect $R_{\mathrm{h}}$ to then asymptotically approach a constant value equal to the radius of the event horizon in $\Lambda \mathrm{CDM}$. What this means, then, is that the current location of $R_{\mathrm{h}}$ affects what we can observe right now, at time $t_{0}$ since the big bang; it is not - and is not meant to be - an indication of how far we will see in our future.

\section{Cosmologies Without Phantom Energy}

We have found it helpful to discuss the properties of null geodesics in FRW cosmologies without resorting to conformal diagrams. As first shown in [13], it is easier for us to think in terms of familiar quantities (proper distances and proper time) that are not always straightforward to interpret otherwise. These authors clearly delineated true horizons from apparent horizons, and extended the definitions, first introduced by [12], in a clear and pedagogical manner.

Using a similar approach, it was shown in [5] that in all cosmologies without phantom energy, all light reaching us today, including that from the recombination region associated with the cosmic microwave background (CMB), has traveled a net proper distance of at most $\sim 0.5 c t_{0}$. Because all causally connected sources in an expanding universe began in a vanishingly small volume as $t \rightarrow 0$, the maximum proper distance from which we receive light today must necessarily be finite (and proportional to $c t_{0}$ ), since presumably there were no pre-existing sources at a non-zero proper distance prior to $t=0$ with which we were in causal contact.

The proof that $R_{\mathrm{h}}(t)$ is the limit to our observability at any time $t$ is quite straightforward, and we reproduce it here for completeness. To begin with, we first derive the equation

\footnotetext{
${ }^{1}$ We won't reproduce the contextual argument here, but instead refer the interested reader to the earlier discussion in [1] and [2].
} 
governing photon trajectories in a cosmology consistent with the FRW metric (Equation 2.1). From the definition of proper distance, we see that

$$
\dot{R}=\dot{a} r+a \dot{r} .
$$

But the null condition for geodesics (see, e.g., [13]) applied to Equation (2.1) yields

$$
c d t=-a(t) \frac{d r}{\sqrt{1-k r^{2}}}
$$

where we have assumed propagation of the photon along a radius towards the origin. The best indications we have today are that the universe is flat so, for simplicity, we will assume $k=0$ in all the calculations described below, and therefore (for a photon approaching us) $\dot{r}=-c / a$. Thus, we can write Equation (3.1) as follows:

$$
\dot{R}_{\gamma}=c\left(\frac{R_{\gamma}}{R_{\mathrm{h}}}-1\right),
$$

where we have added a subscript $\gamma$ to emphasize the fact that this represents the proper distance of a photon propagating towards us. Note that in this expression, both $R_{\gamma}$ and $R_{\mathrm{h}}$ are functions of cosmic time $t$. The gravitational radius must therefore be calculated according to Equation (2.4).

Photons reaching us at $R_{\gamma}\left(t_{0}\right)=0$ attained a maximum proper distance $R_{\gamma}^{\max }$ at time $t_{\max }$ which, according to Equation 3.3, occurred when $\dot{R}_{\gamma}=0$. At this turning point, $R_{\gamma}\left(t_{\max }\right)=R_{\mathrm{h}}\left(t_{\max }\right)$. But for all $w \geq-1$, Equation (2.4) shows that $\dot{R}_{\mathrm{h}} \geq 0$, and therefore $R_{\mathrm{h}}(t) \geq R_{\mathrm{h}}\left(t_{\max }\right)$ for all $t \geq t_{\max }$. It is therefore clear that $R_{\mathrm{h}}(t) \geq R_{\gamma}\left(t_{\max }\right) \equiv R_{\gamma}^{\max }$ for all $t \geq t_{\max }$. Reference [8] contests this conclusion, but without an explanation for why this result is incorrect. In the absence of any evidence to the contrary, one must therefore conclude that $R_{\mathrm{h}}\left(t_{0}\right)$ is indeed the limit to our observability today for any cosmology with $w \geq-1$-and this result stands irrespective of whether this limit may be violated by alternative cosmologies with phantom energy.

\section{Phantom Energy}

The identification of dark energy as a cosmological constant is sometimes called into question because of its very low density compared to expectations of the quantum vacuum. Many workers have attempted to circumvent these difficulties by proposing alternative forms of dark energy, including Quintessence [14,15], which represents an evolving canonical scalar field with an inflation-inducing potential, a Chameleon field (see, e.g., $[16,17,18]$ ) in which the scalar field couples to the baryon energy density and varies from solar system to cosmological scales, and modified gravity, arising out of both string motivated, or General Relativity modified actions $[19,20,21]$, which introduce large length scale corrections modifying the late time evolution of the Universe. The actual number of suggested remedies is far greater than this small, illustrative sample.

Of these, the most unusual alternative cosmology appears to be the one in which dark energy has the equation-of-state parameter $w_{\mathrm{de}}<-1[7,22]$. Dubbed "phantom" energy, only the presence of such a constituent can lead to a Universe in which the overall equationof-state parameter $w$ can also be $<-1$, the situation highlighted in [8] as "proof" that $R_{\mathrm{h}}$ 
does not represent a true horizon, even for universes with the equation-of-state parameter $w \geq-1$, which - as we have just reiterated - is demonstrably incorrect.

Before we consider the null geodesics in this kind of universe, let us first quickly review the properties of a phantom cosmology. The energy conservation equation in general relativity tells us that as the Universe expands,

$$
\dot{\rho}=-3 H(\rho+p)=-3 H \rho(1+w) .
$$

Therefore, if the presence of a phantom energy causes $w<-1$, the overall energy density in the Universe grows with time, which seems counter-intuitive because expansion should actually deplete the energy supply. ${ }^{2}$ Related to this is the fact that the only possible way to obtain $w_{\text {de }}<-1$ is by violating the so-called dominant energy condition (DEC), which for a perfect cosmological fluid may be stated as

$$
\rho_{\mathrm{de}} \geq\left|p_{\mathrm{de}}\right|
$$

where, in obvious notation, $\rho_{\mathrm{de}}$ and $p_{\mathrm{de}}$ refer to the density and pressure, respectively, of the phantom energy field.

The physical motivation for the DEC is to prevent instability of the vacuum or propagation of energy outside the light cone, a situation arising from the fact that the sound speed in such a fluid is greater than $c$. In a finite time after the big bang, both the expansion factor and energy density of the phantom field blow up, leading to a phenomenon known as the "big rip" [22]. Therefore, a cosmology with permanent phantom energy appears to have very little resemblance to what we actually see in nature. Under most optimistic assumptions [23], the instability timescale in such a scenario can be greater than the current age of the Universe, but only in a highly contrived manner.

Nonetheless, there are situations involving phantom energy that do not lead to a big rip, and that could also be consistent with the current data. It is therefore important to understand the behavior of $R_{\mathrm{h}}$, and whether it limits the size of the visible universe, even when $w<-1$. An interesting class of such cosmologies is the Scalar-Tensor Models [24,25], in which the combination of two functions of the field in the Lagrangian, derived from some underlying theory (such as brane cosmology), provides enough flexibility for the Universe to experience a temporary phase of phantom energy, while still crossing the phantom divide line (i.e., $p=-\rho$ ) some time in the future, thereby preventing the otherwise unavoidable big rip.

What would null geodesics look like in a cosmology with phantom energy? Figure 1 shows $R_{\mathrm{h}}(t)$ as a function of cosmic time $t$ (thick black curve) for a $\Lambda$ CDM-like Universe in which the dark energy component is replaced by a phantom field with $w_{\text {de }}=-1.1$. Blending this energy with matter and radiation at early times, such a Universe's overall equation-ofstate parameter $w$ is still $>0$ until $t \sim 0.5 t_{0}$, after which $w_{\text {de }}$ takes over and $w$ drops to negative values. (Note that simply for convenience, we scale all of our quantities here to the current age of the Universe, $t_{0} \approx 13.7 \mathrm{Gyr}$, in the standard model.) It is easy to see from Equation 2.4 that $\dot{R}_{\mathrm{h}}<0$ subsequent to this transition, and therefore $R_{\mathrm{h}} \rightarrow 0$ at a time corresponding to the "big rip" when both $a(t)$ and $\rho(t)$ diverge to infinity.

Several of the null geodesics available in such a cosmology are indicated by the thin curves in this figure. In each case, $R_{\gamma}$ is the proper radius of photons travelling along a given

\footnotetext{
${ }^{2}$ Note that even in de Sitter, one often has difficulty justifying why $\rho$ remains constant during the expansion, though arguments based on properties of the false vacuum can gain some traction. But phantom energy is much worse, since even a false vacuum presumably has a limited energy supply.
} 


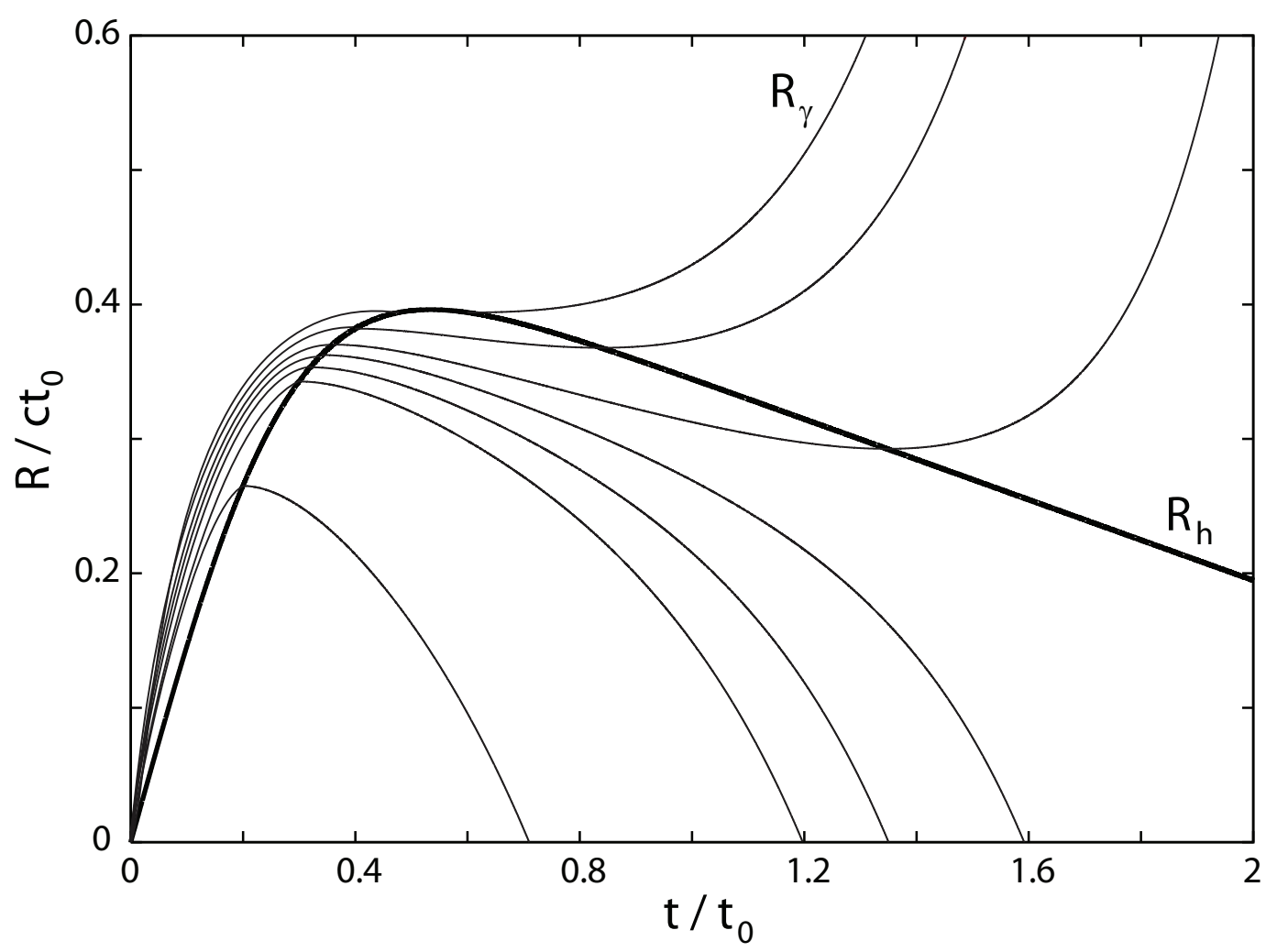

Figure 1. Proper distance $R$ in units of $c t_{0}$ versus $t$ in units of $t_{0}$, where $t_{0}=13.7$ Gyr is the current age of the Universe within the context of the standard model. The thick curve labeled $R_{\mathrm{h}}$ is the gravitational radius of a $\Lambda$ CDM-like universe with dark energy replaced by a phantom field with equation-of-state parameter $w_{\mathrm{de}}=-1.1$. The thin curves represent null geodesics, all of which approach $R_{\gamma}(0)=0$ as $t \rightarrow 0$. In each case, $R_{\gamma}$ is the time-dependent proper radius of photons travelling along a given null geodesic, distinguished by the (chosen) time $t_{\text {max }}$ at which $\dot{R}_{\gamma}=0$ (see Equation 3.3). The most important feature of these curves is that none of those actually reaching us at $R_{\gamma}(t)=0$ (for $t>0$ ) ever attain a proper distance greater than the maximum extent of our cosmic horizon.

null geodesic, distinguished by the value of $t_{\max }$ at which $\dot{R}_{\gamma}=0$ in Equation (3.3). In other words, for any given cosmic time $t$, sources may be distributed throughout the Universe, and therefore the null geodesics passing through those sources all follow different trajectories $R_{\gamma}(t)$ bringing them to the origin $\left(R_{\gamma}=0\right)$, where the observer is located. But for each observation time $t_{0}$, only one null geodesic connects the observer to all the sources emitting light along its trajectory shown in this figure.

Each of these curves, the gravitational horizon and every null geodesic, approaches 0 as $t \rightarrow 0$, as one would expect in a universe where no source existed a finite distance from us prior to the big bang. In addition, in order for us to actually see the light propagating along a given geodesic, that curve must reach $R_{\gamma}(t)=0$ for some time $t>0$. Therefore, according to Equation 3.3, the time at which $R_{\gamma}=R_{\mathrm{h}}$ corresponds to a turning point in $R_{\gamma}(t)$, and is in fact a maximum for the proper distance of every geodesic actually reaching us (just as it was for the non-phantom cosmologies we considered earlier).

Reference [8] argued that the existence of geodesics that reverse paths and re-cross $R_{\mathrm{h}}$ 
violate its function as a horizon. But what they fail to recognize is that every null geodesic that possesses a second turning point (where again $\dot{R}_{\gamma}=0$, corresponding to another time at which $R_{\gamma}=R_{\mathrm{h}}$ in Equation 3.3), diverges to infinity. It never again comes back to the origin where we are located. In other words, it is essential to remember the rather simple notion that in order for us to see light reaching us from distant sources, the null geodesics must actually end at $R_{\gamma}=0$, not at infinity. And the most important feature illustrated by this diagram is that none of the geodesics reaching us at $R_{\gamma}=0$ ever attained a proper distance greater than the maximum extent of our cosmic (or gravitational) horizon.

\section{Concluding Remarks}

We have reaffirmed the physical significance of the Universe's gravitational horizon, which also defines the size of the Hubble sphere. For any non-phantom cosmology, we have proven that its current value, $R_{\mathrm{h}}\left(t_{0}\right)$, is the maximum proper distance to any source producing light in the past that is reaching us today. In a Universe with unbounded energy growth, $R_{\mathrm{h}}$ eventually shrinks to zero when the Universe ends its life in a "big rip." But even in this case, we have demonstrated that no null geodesic reaching us at $R_{\gamma}=0$ will have ever attained a proper distance greater than the maximum extent of $R_{\mathrm{h}}$. When $w \geq-1, R_{\mathrm{h}}$ grows indefinitely, and therefore its maximum extent simply happens to be its value today, $R_{\mathrm{h}}\left(t_{0}\right)$. When $w<-1$, however, $R_{\mathrm{h}}$ shrinks at late times and its maximum extent therefore occurred in our past. Under no circumstances will any null geodesic ever reach us after having propagated to proper distances exceeding our cosmic horizon.

\section{Acknowledgments}

This research was partially supported by ONR grant N00014-09-C-0032 at the University of Arizona. I am grateful to Amherst College for its support through a John Woodruff Simpson Lectureship. I am also grateful to the anonymous referee for suggestions that have improved the manuscript.

\section{References}

[1] Melia, F., "The Cosmic Horizon," MNRAS, 382, 1917 (2007)

[2] Melia, F. \& Shevchuk, A.S.H., "The $R_{\mathrm{h}}=$ ct Universe," MNRAS, 419, 2579 (2012)

[3] Davis, T. M. \& Lineweaver, C. H., "Expanding Confusion: Common Misconceptions of Cosmological Horizons and the Superluminal Expansion of the Universe," Pubs. Astron. Soc. Australia, 21, 97 (2004)

[4] van Oirschot, P., Kwan, J. \& Lewis, G. F., "Through the looking glass: why the 'cosmic horizon' is not a horizon," MNRAS, 404, 1633 (2010)

[5] Bikwa, O., Melia, F. \& Shevchuk, A., "Photon geodesics in Friedmann-Robertson-Walker cosmologies," MNRAS, 421, 3356 (2012)

[6] de Sitter, W., "On the relativity of inertia: Remarks concerning Einstein's latest hypothesis," Proc. Akad. Wetensch. Amsterdam, 19, 1217 (1917)

[7] Caldwell, R. R., "A phantom menace? Cosmological consequences of a dark energy component with super-negative equation of state," Phys. Lett. B, 545, 23 (2002)

[8] Lewis, G. F. \& van Oirschot, P., "How does the Hubble sphere limit our view of the Universe?" MNRAS Letters, 423, 26 (2012) 
[9] Melia, F., "Constraints on Dark Energy from the Observed Expansion of our Cosmic Horizon," Int. J. Mod. Phys. D, 18, 1113 (2009)

[10] Melia, F. and Abdelqader, M., "The Cosmological Spacetime," IJMP-D, 18, 1889 (2009)

[11] Birkhoff, G., Relativity and Modern Physics (Cambridge: Harvard University Press) (1923)

[12] Rindler, W., "Visual horizons in world models," MNRAS, 116, 662 (1956)

[13] Ellis, G.F.R. \& Rothman, T., "Lost horizons," Am. J. Phys. 61, 883 (1993)

[14] Ratra, B. \& Peebles, "Cosmological consequences of a rolling homogeneous scalar field," J., Phys. Rev. D, 37, 3406 (1988)

[15] Wetterich, C., "Cosmology and the fate of dilatation symmetry," Nucl. Phys. B, 302, 668 (1988)

[16] Brax, P., van de Bruck, C., Davis, A.-C., Khoury, J., \& Weltman, A., "Detecting dark energy in orbit: The cosmological chameleon," Phys. Rev. D., 70, 123518 (2004)

[17] Khoury, J. \& Weltman, A., "Chameleon Fields: Awaiting Surprises for Tests of Gravity in Space," Phys. Rev. Lett., 93, 171104 (2004)

[18] Mota, D. F. \& Barrow, J. D., "Local and global variations of the fine-structure constant," MNRAS, 349, 291 (2004)

[19] Capozziello, S., Cardone, V. F., Carloni, S. \& Troisi, A., "Curvature Quintessence Matched with Observational Data," IJMP-D, 12, 1969 (2003)

[20] Nojiri, S. \& Odintsov, S. D., "Modified gravity with negative and positive powers of curvature: Unification of inflation and cosmic acceleration," Phys. Rev. D, 68, 123512 (2003)

[21] Carroll, S. M., Duvvuri, V., Trodden, M. \& Turner, M. S., "Is cosmic speed-up due to new gravitational physics?" Phys. Rev. D, 70, 043528 (2004)

[22] Caldwell, R. R., Kamionkowski, M. \& Weinberg, N. N., "Phantom Energy: Dark Energy with wi-1 Causes a Cosmic Doomsday," Phys. Rev. Lett., 91, Issue 7, id. 071301 (2003)

[23] Carroll, S. M., Hoffman, M. \& Trodden, M., "Can the dark energy equation-of-state parameter w be less than -1?" Phys. Rev. D, 68, Issue 2, id. 023509 (2003)

[24] Boisseau, B., Esposito-Farese, G., Polarski, D. \& Starobinsky, A. A., "Reconstruction of a Scalar Tensor Theory of Gravity in an Accelerating Universe," Phys. Rev. Lett., 85, 2236 (2000)

[25] Gannouji, R., Polarski, D., Ranquet, A. \& Starobinsky, A. A., "Scalar-Tensor Models of Normal and Phantom Dark Energy," JCAP, 0609, id. 016 (2006) 